\title{
Transfusion of minor histocompatibility antigen-mismatched platelets induces rejection of bone marrow transplants in mice
}

\author{
Seema R. Patel, Chantel M. Cadwell, Arielle Medford, and James C. Zimring
}

Center for Transfusion and Cellular Therapies, Department of Pathology and Laboratory Medicine, Emory University School of Medicine, Atlanta, Georgia, USA.

\begin{abstract}
Bone marrow transplantation (BMT) represents a cure for nonmalignant hematological disorders. However, compared with the stringent conditioning regimens used when performing BMT to treat hematological malignancies, the reduced intensity conditioning regimen used in the context of nonmalignant hematological disorders leads to substantially higher rates of BMT rejection, presumably due to an intact immune system. The relevant patient population typically receives transfusion support, often including platelets, and the frequency of BMT rejection correlates with the frequency of transfusion. Here, we demonstrate that immunity to transfused platelets contributes to subsequent BMT rejection in mice, even when the BMT donor and recipient are MHC matched. We used MHC-matched bone marrow because, although immunity to transfused platelets is best characterized in relation to HLA-specific antibodies, such antibodies are unlikely to play a role in clinical BMT rejection that is HLA matched. However, bone marrow is not matched in the clinic for minor histocompatibility antigens, such as those carried by platelets, and we report that transfusion of minor histocompatibility antigen-mismatched platelets induced subsequent BMT rejection. These findings indicate previously unappreciated sequelae of immunity to platelets in the context of transplantation and suggest that strategies to account for minor histocompatibility mismatching may help to reduce the chance of BMT rejection in human patients.
\end{abstract}

\section{Introduction}

Patients who have bone marrow failure syndromes are typically given supportive care, including transfusions of rbc and platelets (PLTs). Some marrow failure patients subsequently undergo bone marrow transplantation (BMT) in an effort to restore endogenous hematopoiesis (1-4). In general, rejection of a BMT is now a rare event due to the use of stringent conditioning regimens that substantially decrease recipient immunity. However, such regimens have considerable toxicity that can lead to morbidity and mortality. This toxicity is an acceptable risk when treating a patient with a malignancy, as the toxic effects have the benefit of killing cancerous cells in addition to promoting engraftment. However, for patients receiving BMT for marrow failure syndromes that do not involve malignancy, the toxic side effects of stringent conditioning regimens are difficult to justify. Accordingly, reduced intensity conditions have been developed to allow BMT without substantial toxicity $(3,5,6)$. However, engraftment is less efficient under these conditions; up to $15 \%$ of transplanted patients reject the $\operatorname{BMT}(4,7,8)$, with a particular increase in rejection in patients with aplastic anemia.

Several causes for the increased BMT rejection rates under reduced intensity regimens have been proposed, one of which is immunological rejection of transplanted marrow. It has been noted that the frequency of BMT rejection correlates to the number of transfusions received $(7,9-13)$, and PLTs are routinely given as part of transfusion support to some marrow failure patients. Clinical concern regarding immune responses to PLT transfusions is typically focused on the development of HLA antibodies and consequent refractoriness to subsequent PLT transfusions. HLA antibodies are less likely to play a role in BMT

Conflict of interest: J.C. Zimring has received a research grant unrelated to the current work from Immucor Inc.

Citation for this article: J. Clin. Invest. 119:2787-2794 (2009). doi:10.1172/JCI39590. rejection, especially in matched siblings in whom HLAs are essentially identical. However, in nonsiblings, because HLA matching is seldom perfect, HLA antibodies may still contribute to BMT rejection. In contrast with HLA, bone marrow is not matched for minor histocompatibility antigens (mHAs). mHAs are polymorphic proteins that differ among individuals by one or more amino acids (14-16) and can elicit humoral responses to their native structure or $\mathrm{T}$ cell responses when recipient MHC presents peptides containing the variant amino acids.

Traditionally, leukocytes in transfused blood products have been thought to be the main source of immunization. Accordingly, PLT products are now routinely stringently leukoreduced prior to transfusion; indeed, removal of leukocytes substantially decreases humoral HLA alloimmunization (17-19). However, human PLTs also carry serologically defined human PLT antigens (HPAs) (20), which consist of amino acid polymorphisms that may themselves be presented in recipient MHC. Thus, it is possible that the transfused PLTs themselves are a source of mHA sensitization. To test this hypothesis, we developed methodologies for collecting, filter leukoreducing, and transfusing murine PLTs in a fashion that models human PLT transfusion. Using this approach, we tested the hypothesis that transfusion of leukoreduced PLT (LR-PLT) concentrates primes recipients for rejection of subsequent MHCmatched BMT across mHA barriers. We developed an MHCmatched:mHA-mismatched BMT model in a murine system, using reduced intensity conditioning. Rejection was observed in recipients transfused with mHA-disparate LR-PLT products, whereas engraftment was observed in recipients that were naive or transfused with syngeneic C57BL/6 (B6) LR-PLTs. These findings demonstrate that PLT transfusions are capable of inducing rejection of MHC-matched BMT across mHA differences and reveal previously unappreciated potential immunological sequelae of PLT transfusion in the context of transplantation. 
A

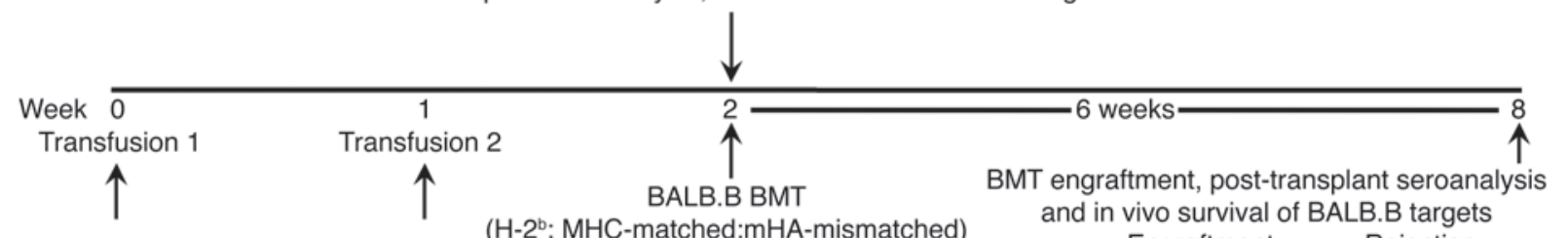

( $\mathrm{H}-2^{\mathrm{b}}$; MHC-matched:mHA-mismatched) and in vivo survival of BALB.B targets
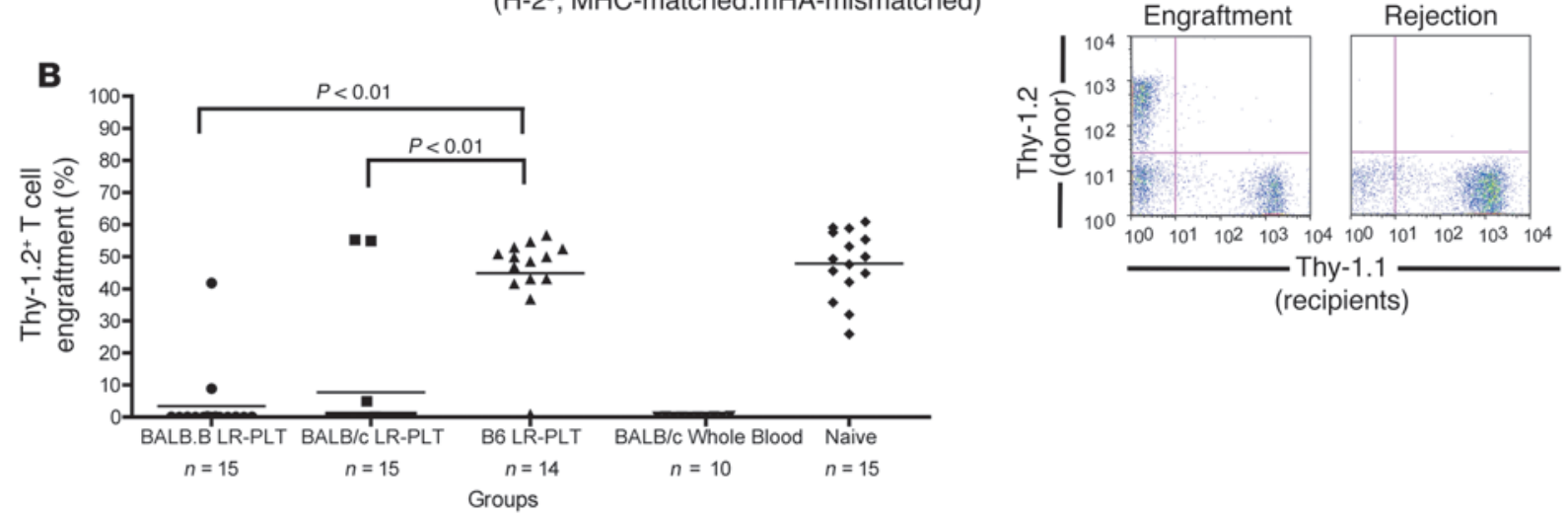

\section{Figure 1}

Transfusion of LR-PLT products induces MHC-matched BMT rejection. (A) Experimental model of MHC-matched BMT. All recipients were B6 Thy1.1 mice $\left(\mathrm{H}-2^{\mathrm{b}}\right)$. BALB.B donors were $\mathrm{MHC}$-matched:mHA-mismatched, while BALB/c donors were MHC- and mHA-mismatched. Groups were as follows: (a) BALB.B LR-PLT (H-2 ${ }^{\text {b; }}$ MHC-matched; mHA-mismatched); (b) BALB/c LR-PLT (H-2d; MHC- and mHA-mismatched); (c) C57BL/6J LR-PLT (H-2b; MHC- and mHA-matched); (d) BALB/c whole blood (H-2d; MHC- and mHA-mismatched); (e) naive. Indicated recipients received 2 PLT transfusions followed by BMT. Seroanalyses and in vivo survival of BALB targets was also performed both prior to and after BMT (see Figures 3 and 4). (B) BMT engraftment results. Engraftment is represented by percentage of Thy $1.2^{+}$cells of total T cells in peripheral blood; the mean of each group is represented as a horizontal line. Statistics were generated using 1-way ANOVA with Dunnett's post test. Combined data from 3 independent experiments are shown.

\section{Results}

Transfusion of LR-PLT products induces BMT rejection. To test the hypothesis that transfusions of LR-PLT products induce rejection of MHC-matched BMT across MHA differences, an MHCmatched:mHA-mismatched BMT model was developed (Figure 1A). In this system, B6 Thy $1.1\left(\mathrm{H}-2^{\mathrm{b}}\right)$ recipients were transfused twice with BALB.B (H-2 ; mHA-mismatched) or BALB/c $\left(\mathrm{H}-2^{\mathrm{d}}\right.$; MHC- and mHA-mismatched) LR-PLT concentrates. One week after the second transfusion, recipients received a BALB.B BMT under reduced intensity conditions. Six weeks later, BMT engraftment was assessed using the $\mathrm{T}$ cell congenic markers Thy 1.1 (recipient) and Thy1.2 (donor). All donors and recipients were females. Engraftment was defined as a percentage of donor cells that exceeded 2 SDs above the mean of positive control mice that were known to reject (transfused with $\mathrm{BALB} / \mathrm{c}$ whole blood). Because the recipients and BM donors were MHC-matched in this system, alloreactivity against MHC is not available as a rejection vector. In contrast, rejection across mHA barriers remains possible due to mHA mismatches between donors and recipients. As the BM and PLT donors are on the same genetic background and thus express the same mHAs, BMT rejection due to sensitization against mHAs in LR-PLT products is possible.

The combined results of 3 experiments demonstrated that 13 of $15(86 \%)$ of the recipients transfused with BALB.B LR-PLT products rejected the BALB.B BMT (Figure 1B). We hypothesized that immunization to mHAs occurred through processing and presentation of donor BALB antigens by recipient APCs (indirect pathway). However, as BALB.B donors and B6 Thy1.1 recipients both express $\mathrm{H}-2^{\mathrm{b}} \mathrm{MHC}$, direct presentation by donor cells may also have occurred. To test the requirement for direct presentation, BALB/c PLT donors were used. Since the BALB/c LR-PLT concentrates were MHC mismatched to the recipients (Figure 1B), direct presentation of BALB mHAs to recipient lymphocytes in the context of the recipients' self $\mathrm{MHC}\left(\mathrm{H}-2^{\mathrm{b}}\right)$ was not available. 12 of 15 (80\%) of the recipients transfused with $\mathrm{BALB} / \mathrm{c}$ LR-PLT concentrates rejected the BALB.B BMT (Figure 1B), suggesting that indirect presentation of mHAs was sufficient to induce rejection of the BMT.

The observed rejection was not due to insufficient recipient conditioning and/or lack of bone marrow viability, as the naive recipients uniformly engrafted the BALB.B BMT (Figure 1B). Likewise, rejection was not due to nonspecific effects of the PLT transfusion process, since engraftment was observed in 13 of 14 (93\%) of the recipients receiving syngeneic (B6) LR-PLT products (Figure 1B); the single mouse that did not have engraftment also did not have anti-BALB immunity after transplant (see below) and thus likely represents failure of the BMT injection, not immunological rejection. Together, these findings demonstrate that LR-PLT product transfusions induce rejection of MHCmatched BMT across mHA barriers.

Assessment of murine LR-PLT concentrates. Although PLT isolation procedures vary among the United States, Canada, and Europe, most units are passed over leukoreduction filters to remove the vast majority of leukocytes. To model this process in our murine system, mouse blood was centrifuged at a slow speed, PLT-rich plasma (PRP) was isolated by collecting the supernatant (as is the method used in the United States), and the PRP was then passed over the brand of leukoreduction filters used to process human PLTs (Pall Corp.). To assess the quality of murine PLT products processed by 

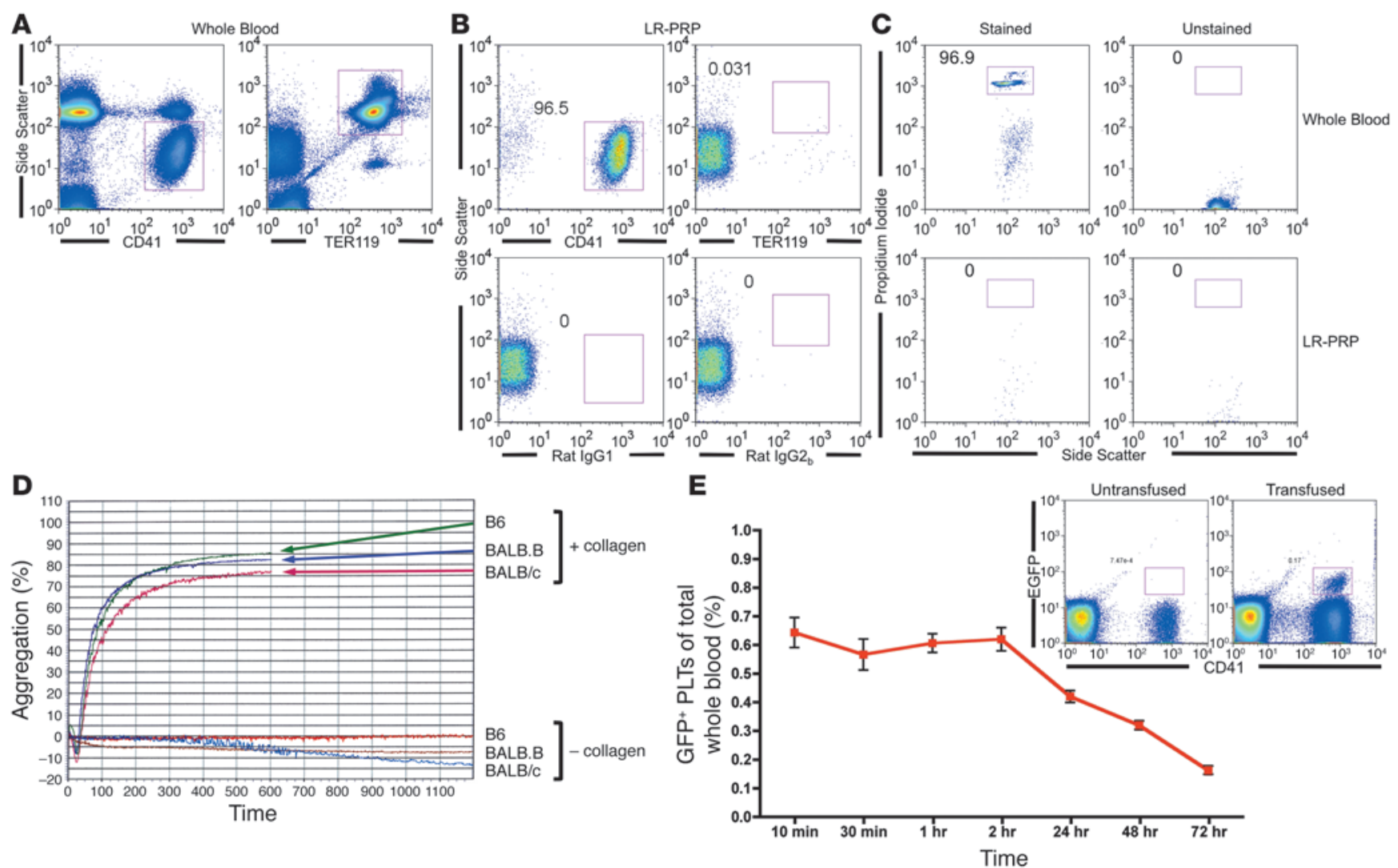

\section{Figure 2}

Quality of isolated LR-PLT concentrates. (A-C) The quantity of PLTs, rbc, and leukocytes was assessed using cell surface markers CD41 and TER119 (A and B) or nucleic acid binding PI (C), respectively. Isotype controls (rat IgG1 for PLTs and rat IgG2 for rbc) and unstained samples were also enumerated to control for nonspecific signal (B and $\mathbf{C}$, respectively). Whole blood samples ( $\mathbf{A}$ and $\mathbf{C})$ were included for gating purposes. (D) Functionality of isolated PLTs. B6, BALB.B, and BALB/c PLTs were exposed to collagen and measured for percentage of aggregation over time using an aggregometer. PLTs without collagen exposure were included to control for spontaneous aggregation. (E) In vivo survival of LR-PLT products. PLTs from EGFP transgenic mice were transfused into naive B6 recipients and tracked over time. Data represent mean \pm SEM. Representative flow cytometry is presented; the left plot utilizes an untransfused mouse to establish background, while the right plot illustrates EGFP+CD41+ PLTs from a transfused recipient. Parts $\mathbf{A}-\mathbf{C}$ demonstrate the gating strategy used for quantifying each cell subset. Numbers indicate the percentage of the gated population as a function of total events. All panels show representative data from experiments reproduced at least 3 times.

this method, for each preparation that was transfused, PLTs, rbc, and leukocytes were enumerated using lineage-specific markers CD41 (PLTs), TER119 (rbc), or nucleic acid-binding propidium iodide (PI) (nucleated leukocytes) (Figure 2, A-C). Whole blood was used to establish scatter and staining gates for rbc and PLTs (Figure 2A). The composition of the LR-PLT products was approximately 97\% PLTs (Figure 2B), and only low amounts of rbc were observed. Background staining was established using isotype controls and unstained samples (Figure 2B). Leukocyte gates from PI staining were established on whole blood (Figure 2C), and leukocytes were undetectable in all but one LR-PLT transfusion (Figure 2C and Table 1). Titrations of a known concentration of leukocytes in LR-PRP demonstrated that the leukocyte assay detected as few as 500 leukocytes per transfused unit (data not shown). Thus, LR-PLT units contained approximately $1 \times 10^{8}$ PLTs $(200,000 / \mu \mathrm{l}), 5 \times 10^{4}$ $\mathrm{rbc}(100 \mathrm{rbc} / \mu \mathrm{l})$, and fewer than 500 leukocytes $(1 / \mu \mathrm{l}) .1 \times 10^{8}$ PLTs were transfused, as this is an approximate volume adjusted equivalent to the transfusion of a single unit of human apheresis PLTs, which contain an average of $3 \times 10^{11}$ or more PLTs (21).
The quality and functionality of the PLTs themselves was assessed using a PLT aggregometer routinely used in the clinical labs for assessment of PLT function. Upon exposure to a physiological substance (collagen), PLTs in the LR-PLT concentrates aggregated normally (Figure 2D), indicating that the murine LR-PLTs retain the ability to aggregate in response to a natural agonist. Posttransfusion survival of murine LR-PLTs was tested by transfusing into naive $\mathrm{B} 6\left(\mathrm{H}-2^{\mathrm{b}}\right)$ recipients. To allow visualization of the transfused PLTs, donors consisted of transgenic mice expressing EGFP in their PLTs. $\mathrm{EGFP}^{+} \mathrm{CD} 41^{+}$PLTs were easily detectable in the peripheral blood for at least 72 hours after transfusion (Figure 2E), consistent with published values of murine PLT survival after transfusion (22).

PLTs were also assessed using a routine blood bank method, the "swirl" test (23). In this test, the quality of the PLT morphology was determined by the presence or absence of "swirling" when PLTs were gently rocked while held up to a light. Prior to each transfusion, the processed PLTs "swirled" (data not shown), indicating that the PLTs were discoid in shape (23). PLTs with an 


\section{Table 1}

Assessment of leukocyte and rbc contamination in LR-PLT units

$\begin{array}{lcc}\text { Groups } & \text { Average leukocytes per transfusion unit } & \text { Average rbc per transfusion unit } \\ \text { BALB.B LR-PRP } & \text { Undetectable }(n=5) ; 118.6(.24 / \mu \mathrm{l})(n=1) & 5.8 \times 10^{4}(116 \mathrm{rbc} / \mu \mathrm{l}) \\ \text { BALB/C LR-PRP } & \text { Undetectable } & 4.4 \times 10^{4}(88 \mathrm{rbc} / \mu \mathrm{l}) \\ \text { B6 LR-PRP } & \text { Undetectable } & 5.7 \times 10^{4}(114 \mathrm{rbc} / \mu \mathrm{l})(n=5) ; \text { Undetectable }(n=1)\end{array}$

Every preparation of LR-PRP was evaluated for residual leukocyte and rbc contamination, as described in Methods. Data shown represent mean from all 3 experiments $(n=6)$.

altered morphology (i.e., spherical) due to damage or activation do not "swirl" (23).

Together, the above data demonstrate that the processing of murine LR-PLT products by methodologies that model human PLT processing (i.e., differential centrifugation and filter leukocyte reduction) results in a product that is similar to human PLT products with regard to cellular composition, swirling properties, agonist-based aggregation, and posttransfusion survival.

Analysis of BALB-specific immunity in PLT-transfused recipients. Although it has previously been demonstrated that MHC-mismatched PLT transfusions elicit anti-MHC humoral and cellular alloresponses $(24,25)$, it is unclear whether similar responses are induced against mHAs expressed by the transfused PLT products in the MHC-matched setting. To test the hypothesis that the PLT transfusions induced anti-mHA immunity, recipients were analyzed for anti-BALB antibodies and for the ability to eliminate cells expressing the same mHAs as the BALB LR-PLT concentrates. Naive and BALB/c whole blood-transfused recipients were used as negative and positive controls, respectively.
The presence of anti-BALB antibodies was tested for by an indirect immunofluorescence assay using BALB.B PLT and splenocyte (SPL) targets. Using BALB.B SPL targets, we detected an increased assay signal in recipients transfused with BALB LR-PLT products (Figure 3A) compared with control syngeneic (B6) LR-PLT-transfused recipients; however, this difference did not achieve statistical significance. No difference in anti-BALB antibodies was detected using BALB.B PLT targets, which is not due to inability of the assay to detect PLT-bound antibodies, as both BALB.B PLT and BALB.B SPL target bound antibodies were detected using positive control sera. Thus, no significant levels of anti-BALB antibodies were detected in any of the groups prior to BMT.

PLT-induced immunity against BALB mHAs was also tested through an in vivo survival assay using cellular targets expressing the relevant mHAs. As the assay is terminal, representative mice from each group were utilized and injected intravenously with BALB.B SPL targets. As an internal negative control, B6 SPL targets were coinjected along with the BALB.B SPL targets. To allow target visualization and enumeration, BALB.B and B6 SPL targets

\section{Figure 3}

Analysis of alloimmunization against BALB mHAs before BMT. All recipients were $B 6$.Thy 1.1 mice $\left(\mathrm{H}-2^{\mathrm{b}}\right)$. (A) Indirect immunofluorescence staining was used to assess the presence of antiBALB antibodies using BALB.B SPL (white) and PLT (gray) targets. (B) BALB-specific immunity was assessed by in vivo survival of BALB.B SPL targets. Error bars in $A$ represent mean \pm SEM. The mean of each group in $\mathbf{B}$ is represented as a horizontal line. Statistics were generated using 1-way ANOVA with Dunnett's post test. Data shown are the combined data from 3 separate experiments.

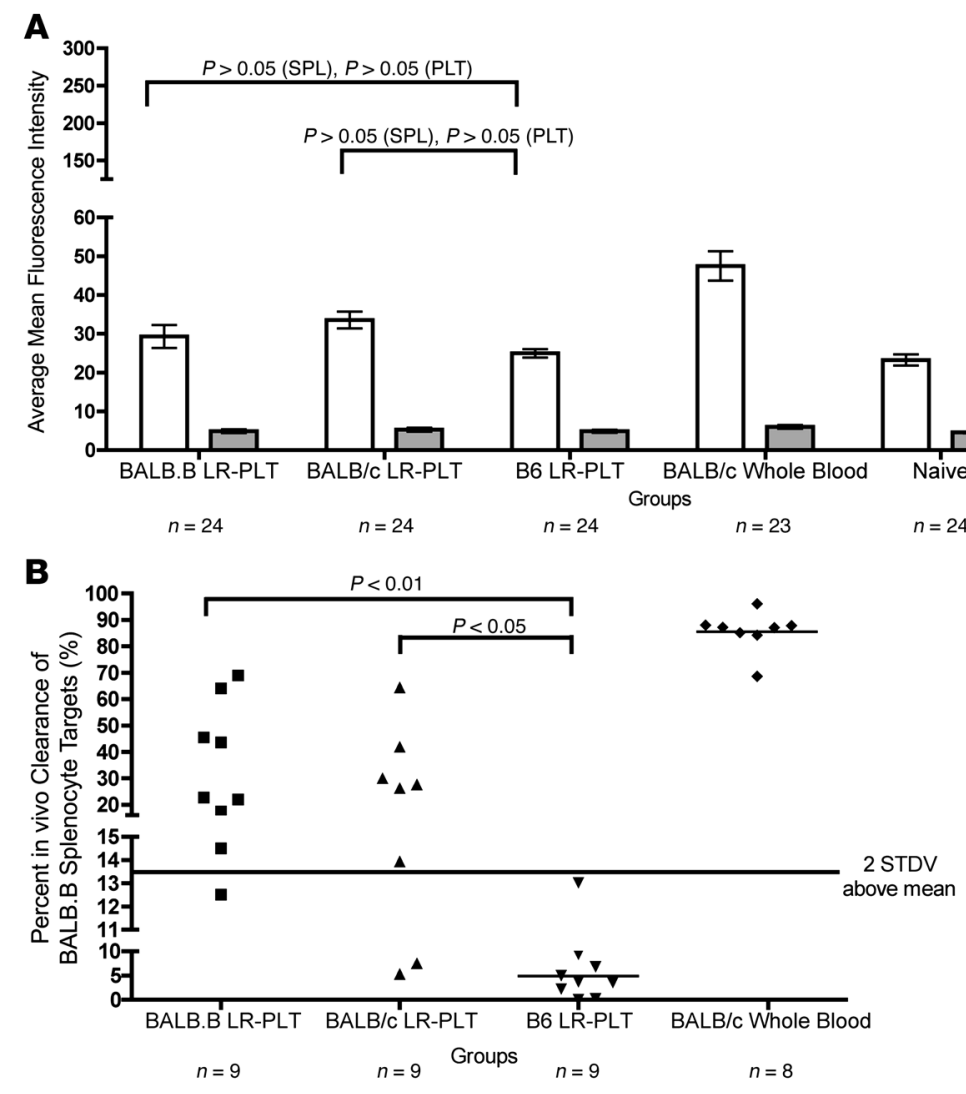




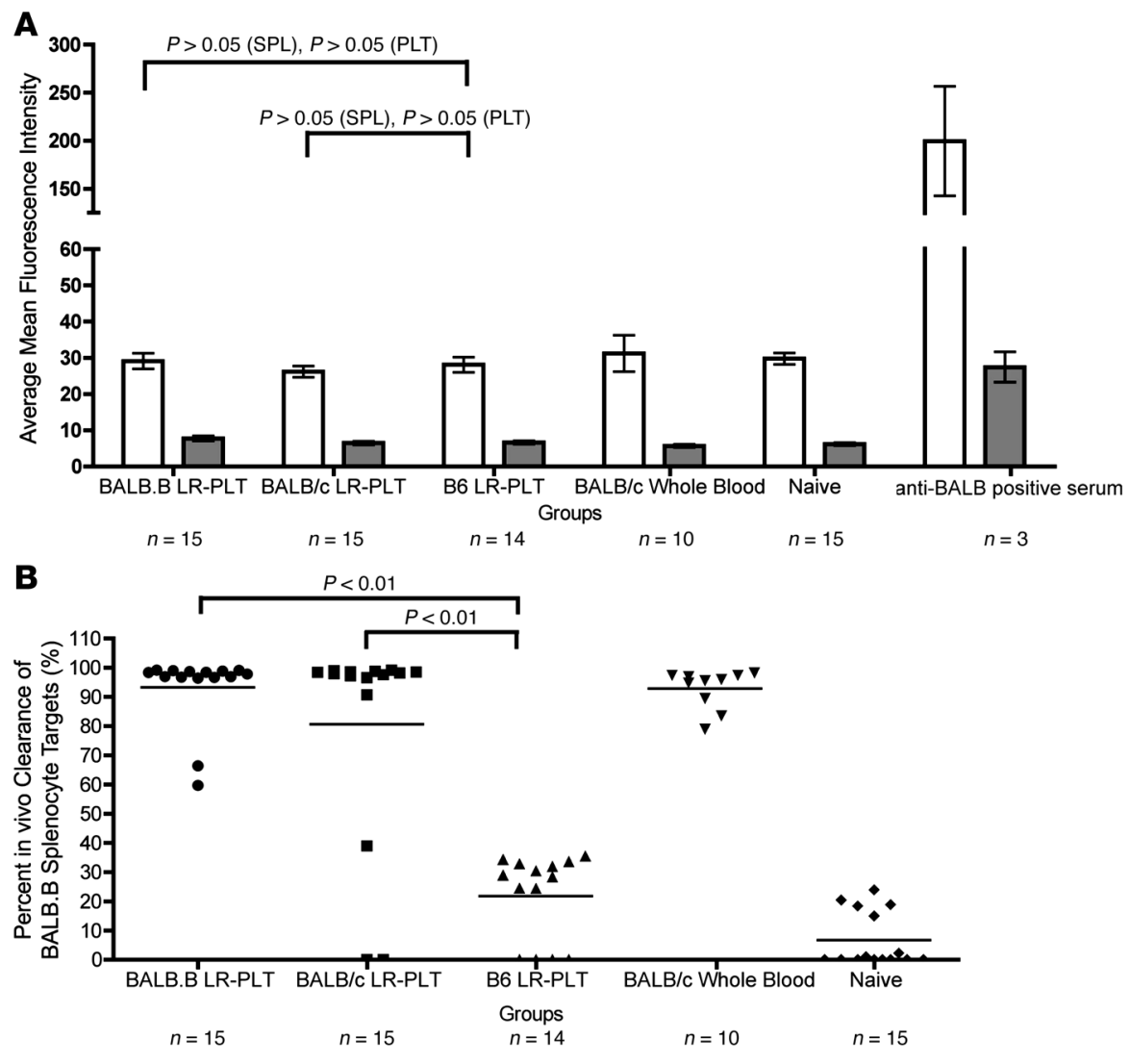

Figure 4

Alloimmunization against BALB mHAs after BMT. All recipients were B6 Thy1.1 mice $\left(\mathrm{H}-2^{\mathrm{b}}\right)$. (A) Indirect immunofluorescence staining was used to assess the presence of anti-BALB antibodies using BALB.B SPL (white) and PLT (gray) targets. (B) BALB-specific immunity was assessed by in vivo survival of BALB.B SPL targets. Error bars in $\mathbf{A}$ represent mean \pm SEM. The mean of each group in $\mathbf{B}$ is represented as a horizontal line. Statistics were generated using 1-way ANOVA with Dunnett's post test. Data shown are combined data from 3 separate experiments.

were labeled with different concentrations of the fluorescent dye CFSE. Twenty-four hours after infusion, recipients were sacrificed and targets were enumerated in the spleen. Numbers of BALB. B SPL targets were normalized to B6 SPL targets to control for differences in injection and splenic processing. Immunity was defined as a specific absence of BALB.B SPL targets (normalized to B6 targets) to an extent that exceeded 2 SDs above the mean of the control mice receiving syngeneic (B6) LR-PLT products. Substantial immunity was observed in recipients transfused with BALB. $\mathrm{B}$ or BALB/c LR-PLT concentrates (8 of 9 [88\%] and 6 of 9 [66\%], respectively) (Figure $3 \mathrm{~B}$ ).

Rejecting recipients demonstrate $B A L B$-specific immunity in vivo in the absence of detectable anti-BALB antibodies. Given that the process of BMT can substantially alter the immune system and responses to transplant antigens, transplanted recipients were tested for BALB mHA-specific immunity after the outcome of BMT was determined (see Figure 1A). Serum was collected to test for antibodies by indirect immunofluorescence (as above); recipients were then injected with CFSE-labeled BALB.B and B6 SPL targets; 24-hour target survival was assessed. No anti-BALB antibody signal was detected above background (naive mice) in any groups, using either SPL or PLT targets (Figure 4A). In contrast, BALB.B SPL targets were eliminated in $100 \%$ of the recipients that had rejected the BMT, with normal target survival in control animals (Figure 4B). The few animals that had received BALB LR-PLTs and did not reject the BMT were the same mice that did not eliminate the BALB.B SPL targets. Of note, the percentage clearance of BALB.B SPL targets observed in 2 of the engrafting recipients previously transfused with BALB.B LR-PLT concentrates was roughly $20 \%-30 \%$ above the background of syngeneic (B6) LR-PLTtransfused recipients (Figures $1 \mathrm{~B}$ and $4 \mathrm{~B}$ ). This raises the possibility that rejection would have subsequently occurred in the 2 engrafting BALB.B LR-PLT-transfused recipients. As the in vivo survival assay is terminal, the ongoing status of the BMT was not further assessed. Together, these data indicate the presence of anti-BALBspecific immunity that correlates with rejection of the BMT. The exact nature of the immunity is unclear, but the data suggest that anti-BALB antibodies are not the effector mechanism.

\section{Discussion}

It has been clinically observed that patients who receive multiple transfusions prior to transplantation have a greater frequency of rejecting HLAmatched BMT under reduced intensity conditioning $(7,9-13)$. Although HLA matching is not perfect except in matched siblings, rejection across HLA barriers is decreased through attempts at HLA matching. However, as no matching is performed for mHAs, mHAs likely represent an additional immune barrier. Because many of the patients are transfused with LR-PLT products as part of their transfusion support, we hypothesize that immune responses to mHAs on the transfused PLTs contribute to the increased rates of BMT rejection by immunizing to mHAs that are also expressed by subsequent stem cells in a BMT. However, the correlation between multiple transfusions and BMT rejection may not be causal, as increased transfusion might simply reflect more advanced disease with greater perturbation of the bone marrow microenvironment. Our data indicate that transfusions of LR-PLTs induce BMT rejection. As the animals have no damage to the bone marrow due to underlying hematological disease, the most likely explanation is rejection induced by LR-PLT transfusions. These findings in no way exclude that when present, bone marrow disease may also contribute to rejection; however, this study does demonstrate that transfusion of mHA-mismatched LR-PLTs is sufficient to induce rejection of MHC-matched BMT with reduced intensity conditioning.

These data do not address the effect of PLT transfusion in the post-BMT setting. Many BMT patients receive multiple units of transfused PLTs as a supportive measure until the transplanted marrow achieves sufficient hematopoiesis. Most patients in this 
situation have received stringent conditioning in the context of neoplasia, resulting in myeloablative outcomes. It is our prediction that PLT transfusion is unlikely to induce BMT rejection in this setting, as the recipient immune system has been largely eliminated; however, this remains to be formally tested. The current studies focus on reduced intensity conditioning regimens in which the recipients' immune system is mostly intact. Moreover, the relevancy of post-BMT PLT transfusion effects is unclear in this setting; mice that reject the BMT remain alive without signs of illness and without PLT transfusion support, indicating that recipient hematopoiesis remains sufficiently intact. Thus, this system models mild conditioning regimens in which post-BMT transfusion support may not be needed.

The transplantation marker utilized in these studies (Thy1.1/ Thy1.2) is a T cell-specific epitope; thus, the engraftment data do not unequivocally rule out the possibility of lineage-specific engraftment. CD45.1/CD45.2 congenic markers, which are expressed broadly on leukocytes, are often used in BMT studies to avoid the issue of lineage-specific engraftment. However, they were avoided in the current studies because it has been reported that CD45.1/CD45.2 can serve as mHAs that contribute to rejection under reduced conditioning regimens (26).

Although substantial PLT enrichment procedures were utilized, there were very low levels of rbc and leukocytes (see Table 1). Since leukocytes can be professional APCs, they could theoretically stimulate recipient T cells as part of the direct pathway. However, when we purposely mismatched the PLT and BMT for MHC while maintaining common mHAs (BALB/c PLT, BALB.B marrow), $B M T$ rejection still occurred at approximately the same frequency (see Figure 1B). We interpret these findings to indicate that the indirect pathway is sufficient to induce BMT rejection. However, one must acknowledge that some $\mathrm{T}$ cell epitopes (e.g., $\mathrm{OVA}_{323-339)}$ can be presented by both $\mathrm{H}-2^{\mathrm{b}}$ and $\mathrm{H}-2^{\mathrm{d}}$ MHCs and T cells generated on one MHC background may cross-react with the other MHC background (27). Thus, although these data suggest indirect presentation, we cannot unequivocally rule out persistence of the direct pathway. However, if this were the case, it is likely that the number of $T$ cells capable of cross-reacting would be substantially fewer and rejection would be less robust. These in vivo findings are consistent with previous reports demonstrating that recipient APCs pulsed in vitro can process and present PLT antigens (25, 28). An additional concern in interpretation is that it is also possible that rejection in the recipients transfused with BALB/c LR-PLT concentrates was due to anti-H- $2^{\mathrm{d}}$ alloresponses cross-reacting with mHA/MHC complexes on the BALB.B BMT. However, we reject this possibility, as we have recently demonstrated that recipients transfused with whole blood from B6 mice congenic for $\mathrm{H}-2^{\mathrm{d}}$ $\left(\mathrm{B} 6 . \mathrm{H}-2^{\mathrm{d}}\right)$, which isolates the anti-H2 ${ }^{\mathrm{d}}$ component, do not reject BALB.B BMT in spite of strong anti-H- $2^{\mathrm{d}}$ alloresponses (29). Thus, while there are alternate interpretations, the most likely implication is that the direct pathway is not required and that the indirect pathway is sufficient to sensitize recipients against mHAs expressed by the transfused PLT products.

Experimentally, the presence of residual $\mathrm{rbc}$ and leukocytes is a concern regarding the identification of the precise transfusion component responsible for inducing rejection. However, this is irrelevant from the standpoint of modeling human transfusion and testing the hypothesis that PLT transfusions induce BMT rejection, as human PLT units have similar contamination with rbc and leukocytes. Indeed, the residual rbc and leukocytes are required to accurately model human PLT transfusions. Thus, the data suggest that transfusions of "PLT units" induce BMT rejection. However, ultimately identifying the exact component responsible will be important in circumventing the problem. For example, if it is indeed indirect presentation of $\mathrm{MHAs}$ on PLTs themselves by recipient APCs that is responsible, then immunomodulation (i.e., immunosuppressants or altered induction regimens) will be required, as one cannot with hold the PLT transfusions due to hemostasis needs. However, if the residual rbc or leukocytes are responsible, then better PLT enrichment procedures may solve the problem. Semple et al. have previously reported that extreme leukoreduced PLTs (from SCID donors) still induce antibody responses (30), consistent with an interpretation that PLTs are alone capable of inducing humoral immunity; however, whether this extends to $\mathrm{CD}^{+} \mathrm{T}$ cell responses is yet to be determined. Thus, in addition to examining the immunological mechanism of rejection, future studies should address the component of the unit responsible for immunization.

The observed rejection occurred in the presence of anti-BALB immunity, as indicated by selective elimination of BALB targets during in vivo target survival assays, which correlated strongly with BMT rejection (see Figures 3 and 4). BALB but not B6 targets were eliminated in the in vivo survival assay. We thus interpret this to be a BALB-specific response. However, it is also possible that a nonspecific change in the recipient biology is induced to which BALB cells are intrinsically more sensitive than B6. Although we cannot unequivocally rule this out, the more likely explanation is that BALB-specific immunity is induced. Such immunity could be either cellular immunity (i.e., cytotoxic T cells) or humoral responses (i.e., antibody-dependent cellular cytotoxicity). However, the absence of detectable anti-BALB antibodies in LR-PLT recipients either before or after BMT (see Figures 3 and 4) suggests that humoral responses are not likely involved. Therefore, the combined findings suggest that cellular immunity against mHAs expressed by transfused LR-PLT concentrates induces rejection of MHC-matched BMT expressing the same mHAs.

Substantial efforts were taken to model processing of human PLT products and assess the quality of the enriched PLTs. To the best of our knowledge, we present a novel model that encompasses human filter leukoreduction, thus closely mimicking the processing of human PLTs. However, one variable of the PLT transfusion process that was not controlled for was the transfusion of stored versus fresh PLTs. Typically, patients receive PLT products that have been stored up to 5 days. When PLTs are stored for a long period of time, they release soluble CD154 (31) and can shed MHC (32). In addition, during storage, residual leukocytes and/or PLTs in the PRP concentrates can release various proinflammatory mediators $(31,33,34)$. In theory, these storage-based changes may alter the immune responses to transfused PLTs.

Currently, the main appreciated immunological sequela of PLT transfusion is the generation of HLA antibodies, which can make patients refractory to subsequent PLT transfusions. The relevancy of the current findings to human medicine is that they suggest an additional immunological vector pertinent to BMT; in particular, PLT transfusion-induced BMT rejection across MHA barriers. It is unclear whether this effect may differ depending upon the cell source for hematopoietic transplantation (i.e., bone marrow, peripheral stem cells, or cord blood). Moreover, while the current studies focus on BMT, to the extent that solid organ transplants share mHAs with PLTs, it is also possible that PLT transfusions promote rejection in other transplant settings. Of course, the cur- 
rent findings are in a mouse model, and testing the hypothesis in a human setting would be required before any clinical conclusions can be drawn. Nevertheless, this raises a potential new issue in transfusion medicine and organ transplantation. Currently, neither mHA disparities nor anti-mHA immunity is measured clinically.

\section{Methods}

Mice. Female C57BL/6J (H-2 $\left.{ }^{\mathrm{b}}\right)$, BALB.B (C.B10-H-2b/LiMcdJ [H-2 $\left.2^{\mathrm{b}}\right]$ ) and $\mathrm{BALB} / \mathrm{c}\left(\mathrm{H}-2^{\mathrm{d}}\right)$ mice were used as donors at 8 to 12 weeks of age. B6.PLThy $1^{a} / \mathrm{CyJ}\left(\mathrm{H}-2^{\mathrm{b}}\right)$ female mice were used as recipients at 6 to 8 weeks of age. Mice were purchased from the Jackson Laboratory. C57BL/6J EGFP mice were a generous gift from Derek A. Persons (St. Jude Children's Research Hospital, Memphis, Tennessee, USA; ref. 35) and were bred by the Emory Division of Animal Resources Animal Husbandry service. All mice were housed in Emory University Department of Animal Resources facilities, and procedures were performed according to protocols approved by the Emory University IACUC committee.

Antibodies for flow cytometry. Antibodies were purchased from BD Biosciences - Pharmingen (anti-CD41 PE, PE anti-TER119/erythroid-PE, rat IgG2 $2_{b}$-PE, APC goat anti-mouse Igs, FITC anti-mouse CD3, PE anti-mouse CD90.2, and FITC anti-mouse CD19) and eBioscience (rat IgG1 and APC anti-mouse CD90.1).

PLT preparation. Donors (C57BL/6J, BALB.B, and BALB/c) were exsanguinated and whole blood was collected in 1:8 acid citrate dextrose (ACD) (Vacutainer; BD). $2 \mathrm{ml}$ of Dulbecco's PBS (DPBS) was added to each $\mathrm{ml}$ of whole blood in a $15-\mathrm{ml}$ conical tube; samples were mixed through gentle inversion, and centrifuged at $80 \mathrm{~g}$ for 10 minutes. PRP was collected, mixed by gentle inversion, and respun at $80 \mathrm{~g}$ for 10 minutes. Isolated PRP was pooled, and approximately $20 \mathrm{ml}$ was passed over a Neonatal Purecell PL High Efficiency Leukocyte Reduction Filter (Pall Corp.). PLTs were enumerated as described below. After enumeration, PLT concentration was normalized to $2 \times 10^{8} \mathrm{PLT} / \mathrm{ml}$ by pelleting at $1942 \mathrm{~g}$ for 10 minutes and resuspending in Tyrode's buffer ( $1 \mathrm{mM}$ HEPES, $137 \mathrm{mM} \mathrm{NaCl}, 2.8 \mathrm{mM}$ $\mathrm{KCl}, 1 \mathrm{mM} \mathrm{MgCl}_{2}, 12 \mathrm{mM} \mathrm{NaHCO}_{3}, 0.4 \mathrm{mM} \mathrm{Na}_{2} \mathrm{HPO}_{4}$, and $5.5 \mathrm{mM}$ glucose in Milli Q water, $\mathrm{pH}$ 7.4). $500 \mu \mathrm{l}$ of resuspended LR-PLT (108 PLT total) was transfused through the tail vein. All PLT handling was performed at room temperature. A "swirl" test was performed on all LR-PRP concentrates to test the quality of PLTs in solution (23).

PLT, rbc, and leukocyte enumeration. To count PLTs and residual rbc, $20 \mu \mathrm{l}$ of LR-PRP was diluted in $100 \mu \mathrm{l}$ FACS buffer (DPBS with $0.2 \%$ BSA and $0.24 \mathrm{mM}$ EDTA) and centrifuged at $1942 \mathrm{~g}$ for 2 minutes at room temperature. The samples were stained with anti-CD41 PE or isotype control rat IgG1 PE for PLTs and anti-TER119 PE or isotype control rat IgG2 2 PE for rbc. All antibodies were used at a dilution of 1:100 in FACS buffer. Samples were incubated in the dark at room temperature for 30 minutes and then washed once with FACS buffer. Samples were resuspended in $100 \mu \mathrm{l}$ FACS buffer. $10 \mu \mathrm{l}$ of each sample was mixed with $940 \mu 1$ FACS buffer, and $2.5 \times 10^{5}$ APC beads (BD Biosciences - Pharmingen) were added to allow counting of absolute numbers. Samples were run on a FACSCalibur and analyzed using FlowJo 8.8.2. 12,500 events of APC-beads were collected, resulting in $50 \mu \mathrm{l}$ of analyzed sample.

Residual leukocytes were enumerated by flow cytometry using the method previously described (36). In brief, a $25 \mu$ l aliquot of LR-PRP was stained using $150 \mathrm{ng} / \mathrm{ml}$ PI in a $1 \mathrm{~g} / \mathrm{l}$ sodium citrate dihydrate solution with $0.7 \%$ Zap-oglobin II reagent (Beckman Coulter) and 0.7\% RNAse cocktail (New England Biolabs), effectively staining all nucleated cells. Nuclei were enumerated on a BD FACSort flow cytometer. A known quantity of APC-labeled beads were added to each sample to allow acquisition of absolute counts (BD Biosciences).

Transfusion of whole blood. Whole blood was collected in 1:8 ACD. The blood was then washed 3 times with mouse PBS to remove excess citrate that is toxic if transfused (37), by centrifuging at $394 \mathrm{~g}$ for 15 minutes. While aspirating the supernatant, the buffy coat was not disrupted. The whole blood was resuspended in $1 \times$ DPBS at a 1:4 dilution, and $500 \mu 1$ transfusions were given by tail vein.

Functionality of LR-PLT products. Prior to transfusion, PLT concentrates in Tyrode's buffer were exposed to collagen and monitored for percentage of aggregation over time using a clinical aggregometer. Aggregation was monitored as a function of light transmission. Tyrode's buffer was used as a blank, and control PLTs without collagen were used to monitor spontaneous aggregation.

In vivo survival of $L R-P L T$ concentrates. Transgenic C57BL/6J female mice expressing EGFP on PLTs were utilized as LR-PLT donors. LR-PLT concentrates were isolated and transfused as above. The presence of EGFP LR-PLTs was measured by enumerating EGFP-positive events in a PLT gate based on forward and side scatter at indicated time points after transfusion. Identity of the events as PLTs was confirmed with anti-CD41 staining at terminal time points.

Seroanalysis. Serum was diluted 1:10 in FACS buffer and incubated with BALB.B SPLs or PLTs for 30 minutes, washed 3 times with FACS buffer, and incubated 30 minutes in a 1:100 dilution of APC goat anti-mouse Igs. B cells were excluded by staining with anti-mouse CD19-FITC (1:50 in FACS buffer), and PLT targets were visualized with anti-mouse CD41 PE (1:100 in FACS buffer). Samples were analyzed by flow cytometry; fluorescence of APC was used to indicate the presence of antibody on targets.

In vivo survival of BALB.B SPL targets. Female C57BL/6J and BALB.B SPLs were isolated and washed in $1 \times$ RPMI, $10 \%$ FBS. SPLs were incubated in rbc lysis buffer (Sigma-Aldrich) ( $2 \mathrm{ml} /$ spleen) for 5 minutes at room temperature. The cells were washed 3 times in $1 \times$ DPBS. During the third wash, cells were enumerated using a hemocytometer. B6 and BALB.B SPL were incubated with $\mathrm{CFSE}^{\text {hi }}(6 \mu \mathrm{M})$ or $\mathrm{CFSE}^{\text {lo }}(5 \mathrm{nM})$, respectively, at $37^{\circ} \mathrm{C}$ for 5 minutes and were then washed. Samples were mixed at equal volumes and injected into tail veins (total dose of $5 \times 10^{6}$ per target).

$B M T$. Bone marrow was harvested from femurs and tibiae by standard techniques. BM cells were enumerated using a hemocytometer and resuspended at $10 \times 10^{6}$ cells $/ \mathrm{ml}$ in $1 \times$ DPBS. $500 \mu \mathrm{l}\left(5 \times 10^{6} \mathrm{BM}\right.$ cells $)$ of BM was injected into the tail veins of all recipients, which were previously conditioned with sublethal gamma irradiation treatments (7 Gy) 24 hours prior to BMT. Engraftment was monitored by staining peripheral blood with anti-CD3 FITC (1:50), anti-Thy1.1 APC (1:2500), and anti-Thy1.2 PE $(1: 2500)$ in FACS buffer for 30 minutes at $4^{\circ} \mathrm{C}$. The cells were washed 3 times and analyzed by flow cytometry. Engraftment was assessed 6 weeks after BMT by percentage donor Thy $1.2^{+} \mathrm{T}$ cells.

Statistics. Statistical analysis was performed using 1-way ANOVA with Dunnett's post test and column statistics. Significance was measured as $P<0.05$.

\section{Acknowledgments}

We thank Krista Hostetler for excellent technical assistance with platelet aggregometry. These studies were supported in part by NIH grant R01HL092977 (to J.C. Zimring).

Received for publication April 17, 2009, and accepted in revised form June 10, 2009.

Address correspondence to: James C. Zimring, Center for Transfusion and Cellular Therapies, Department of Pathology and Laboratory Medicine, Emory University School of Medicine, Woodruff Memorial Building Suite 7107, 101 Woodruff Circle, Atlanta, Georgia 30322, USA. Phone: (404) 712-2174; Fax: (404) 727-5764; E-mail: jzimrin@emory.edu. 
1. Or, R., et al. 2004. Allogeneic stem cell transplantation for the treatment of diseases associated with a deficiency in bone marrow products. Springer Semin. Immunopathol. 26:133-142.

2. Wagner, J.E., et al. 2007. Unrelated donor bone marrow transplantation for the treatment of Fanconi anemia. Blood. 109:2256-2262.

3. Horan, J.T., Liesveld, J.L., Fenton, P., Blumberg, N., and Walters, M.C. 2005. Hematopoietic stem cell transplantation for multiply transfused patients with sickle cell disease and thalassemia after lowdose total body irradiation, fludarabine, and rabbit anti-thymocyte globulin. Bone Marrow Transplant. 35:171-177.

4. Bhatia, M., and Walters, M.C. 2008. Hematopoietic cell transplantation for thalassemia and sickle cell disease: past, present and future. Bone Marrow Transplant. 41:109-117.

5. Resnick, I.B., Shapira, M.Y., and Slavin, S. 2005 Nonmyeloablative stem cell transplantation and cell therapy for malignant and non-malignant diseases. Transpl. Immunol. 14:207-219.

6. Srinivasan, R., et al. 2006. Overcoming graft rejection in heavily transfused and allo-immunised patients with bone marrow failure syndromes using fludarabine-based haematopoietic cell transplantation. Br. J. Haematol. 133:305-314.

7. Champlin, R.E., et al. 2007. Bone marrow transplantation for severe aplastic anemia: a randomized controlled study of conditioning regimens. Blood. 109:4582-4585.

8. Viollier, R., et al. 2008. Recent improvement in outcome of unrelated donor transplantation for aplastic anemia. Bone Marrow Transplant. 41:45-50.

9. Champlin, R.E., et al. 1989. Graft failure following bone marrow transplantation for severe aplastic anemia: risk factors and treatment results. Blood. 73:606-613

10. Deeg, H.J., et al. 1986. Decreased incidence of marrow graft rejection in patients with severe aplastic anemia: changing impact of risk factors. Blood. 68:1363-1368.

11. Gluckman, E., et al. 1992. Bone marrow transplantation for severe aplastic anemia: influence of conditioning and graft-versus-host disease prophylaxis regimens on outcome. Blood. 79:269-275.

12. Stucki, A., et al. 1998. Decreased rejection and improved survival of first and second marrow transplants for severe aplastic anemia (a 26-year retrospective analysis). Blood. 92:2742-2749.

13. Storb, R., et al. 1980. Marrow transplantation in thirty "untransfused" patients with severe aplastic anemia. Ann. Intern. Med. 92:30-36.

14. Goulmy, E. 1996. Human minor histocompatibility antigens. Curr. Opin. Immunol. 8:75-81.

15. Simpson, E., et al. 2002. Minor $\mathrm{H}$ antigens: genes and peptides. Transpl. Immunol. 10:115-123.

16. Roopenian, D.C. 1992. What are minor histocompatibility loci? A new look at an old question. Immunol. Today. 13:7-10.

17. Seftel, M.D., et al. 2004. Universal prestorage leukoreduction in Canada decreases platelet alloimmunization and refractoriness. Blood. 103:333-339.

18. The Trial to Reduce Alloimmunization to Platelets Study Group. 1997. Leukocyte reduction and ultraviolet B irradiation of platelets to prevent alloimmunization and refractoriness to platelet transfusions. N. Engl. J. Med. 337:1861-1869.

19. Schiffer, C.A., et al. 1983. A randomized trial of leukocyte-depleted platelet transfusion to modify alloimmunization in patients with leukemia. Blood. 62:815-820.

20. Rozman, P. 2002. Platelet antigens. The role of human platelet alloantigens (HPA) in blood transfusion and transplantation. Transpl. Immunol. 10:165-181.

21. Roback, J.D., Combs M.R., Grossman, B.J., and Hillyer, C.D. 2008. Technical manual. 16th edition. American Association of Blood Banks. Bethesda, Maryland, USA. 582 pp.

22. Hoffmeister, K.M., et al. 2003. The clearance mechanism of chilled blood platelets. Cell. 112:87-97.

23. Stroncek, D.F., and Rebulla, P. 2007. Platelet transfusions. Lancet. 370:427-438.

24. Sayeh, E., Sterling, K., Speck, E., Freedman, J., and Semple, J.W. 2004. IgG antiplatelet immunity is dependent on an early innate natural killer cellderived interferon-gamma response that is regulated by CD8+ T cells. Blood. 103:2705-2709.

25. Semple, J.W., Speck, E.R., Milev, Y.P., Blanchette, V., and Freedman, J. 1995. Indirect allorecognition of platelets by $\mathrm{T}$ helper cells during platelet transfusions correlates with anti-major histocompatibility complex antibody and cytotoxic $\mathrm{T}$ lymphocyte formation. Blood. 86:805-812.

26. van Os, R., et al. 2001. Immunogenicity of Ly5 (CD45)-antigens hampers long-term engraftment following minimal conditioning in a murine bone marrow transplantation model. Stem Cells. 19:80-87.

27. Robertson, J.M., Jensen, P.E., and Evavold, B.D. 2000. DO11.10 and OT-II T cells recognize a Cterminal ovalbumin 323-339 epitope. J. Immunol. 164:4706-4712.

28. Bang, K.W., Speck, E.R., Blanchette, V.S., Freedman, J., and Semple, J.W. 2000. Unique processing pathways within recipient antigen-presenting cells determine IgG immunity against donor platelet MHC antigens. Blood. 95:1735-1742.

29. Desmarets, M., Cadwell, C.M., Peterson, K.R., Neades, R. and Zimring, J.C. Minor histocompatibility antigens on transfused leukoreduced units of red blood cells induce bone marrow transplant rejection in a mouse model. Blood. Online publication ahead of print. doi:10.1182/blood-200904-214387.

30. Semple, J.W., et al. 1999. Extreme leukoreduction of major histocompatibility complex class II positive B cells enhances allogeneic platelet immunity. Blood. 93:713-720.

31. Blumberg, N., Gettings, K.F., Turner, C., Heal, J.M., and Phipps, R.P. 2006. An association of soluble CD40 ligand (CD154) with adverse reactions to platelet transfusions. Transfusion. 46:1813-1821.

32. Aslam, R., Speck, E.R., Kim, M., Freedman, J., and Semple, J.W. 2008. Transfusion-related immunomodulation by platelets is dependent on their expression of MHC Class I molecules and is independent of white cells. Transfusion. 48:1778-1786.

33. Wadhwa, M., et al. 2000. Cytokines in WBCreduced apheresis PCs during storage: a comparison of two WBC-reduction methods. Transfusion. 40:1118-1126.

34. Bubel, S., Wilhelm, D., Entelmann, M., Kirchner, H., and Kluter, H. 1996. Chemokines in stored platelet concentrates. Transfusion. 36:445-449.

35. Dominici, M., et al. 2005. Transgenic mice with pancellular enhanced green fluorescent protein expression in primitive hematopoietic cells and all blood cell progeny. Genesis. 42:17-22.

36. Hendrickson, J.E., et al. 2006. Recipient inflammation affects the frequency and magnitude of immunization to transfused red blood cells. Transfusion. 46:1526-1536.

37. Zimring, J.C., et al. 2005. Nonhemolytic antibodyinduced loss of erythrocyte surface antigen. Blood. 106:1105-1112. 